\title{
Nota Científica / Short Communication \\ Polinização de Victoria amazonica (Nymphaeaceae) por besouros em condições ex situ no Jardim Botânico Municipal de Bauru/SP
}

\author{
Pollination of Victoria amazonica (Nymphaeaceae) by beetles \\ in ex situ conditions in the Municipal Botanical Garden of Bauru/SP
}

Viviane Camila de Oliveira ${ }^{1,2} \&$ Luiz Carlos de Almeida Neto ${ }^{1}$

\begin{abstract}
Resumo
Pouco se sabe sobre a ecologia de Victoria amazonica fora de sua área de distribuição nativa e faltam estudos sobre a sua reprodução e manejo em condições ex situ, por isso o objetivo deste estudo foi determinar a fauna de besouros presos dentro de flores de V. amazonica cultivadas no Jardim Botânico Municipal de Bauru/ SP e avaliar a eficiência destes como polinizadores. Foi realizada observação focal dos besouros presos nas flores e avaliada a produção de frutos e viabilidade das sementes. Foram encontrados besouros Cyclocephala epistomalis Bates, 1888 presos dentro da câmara floral, sendo o primeiro registro desta espécie no estado de São Paulo. A formação de frutos ocorreu apenas nas flores que apresentaram besouros presos dentro da câmara floral, sendo que das 20 flores observadas, apenas 10 tinham besouros e 9 produziram frutos viáveis com 335,88 sementes em média, com 88\% de taxa de germinação. Em razão do comportamento dos besouros, do grande número de sementes por fruto e elevada taxa de germinação, pode-se inferir que os besouros comportaram-se como polinizadores efetivos.

Palavras-chave: besouros, conservação ex situ, polinização, Victoria amazonica.
\end{abstract}

\begin{abstract}
Little is known about the ecology of Victoria amazonica outside its native area and there is lack of studies on its reproduction and management in ex situ conditions, so the aim of this study was to determine the fauna of beetles trapped inside flowers of $V$. amazonica grown in the Municipal Botanical Garden of Bauru/SP and evaluate the efficiency of these as pollinators. Beetles inside the flowers were monitored through focal observation, and fruit production efficiency and seed viability were tested. Beetles Cyclocephala epistomalis Bates, 1888 were found trapped inside the floral chambers, being the first record of this species in the state of São Paulo. The fruit production occurred only in the flowers that had beetles trapped inside the floral chambers, and of the 20 flowers observed, only 10 had beetles and 9 produced viable fruits with 335.88 seeds on average, hich exhibited $88 \%$ of germination rate. Due to the behavior of the beetles, higher number of seeds per fruit and high germination rate, it can be inferred that the beetles behaved as effective pollinators.
\end{abstract}

Key words: beetles, ex situ conservation, pollination, Victoria amazonica.

\section{Introdução}

A família Nymphaeaceae inclui atualmente oito gêneros e 70 espécies amplamente distribuídas pelo mundo. No Brasil, são encontrados dois gêneros e 23 espécies, dentre as quais destaca-se a Victoria amazonica (Poepp.) J.C. Sowerby (BFG 2015). Esta espécie apresenta grande importância ecológica, medicinal e alimentícia, além da exuberância de suas flores e folhas, tornandose uma das plantas amazônicas mundialmente conhecidas (Rosa-Osman et al. 2011). Victoria amazonica é caracterizada por ervas aquáticas, anuais ou perenes, fixas no substrato, apresentando grandes folhas peltadas flutuantes (Prance \& Arias 1975). A espécie tem como provável centro de origem a região equatorial da bacia do Rio

\footnotetext{
Jardim Botânico Municipal de Bauru, Seção de Coleções Vegetais, Rod. Com. João Ribeiro de Barros (SP-225), km 232, 17035-245, Bauru, SP, Brasil.

${ }^{2}$ Autor para correspondência: vivicamila@yahoo.com.br
} 
Amazonas, ocorrendo em águas calmas com temperaturas em torno de 26 a $30{ }^{\circ} \mathrm{C}$ (Prance 1974; Pott \& Pott 2000; Judd et al. 2002). Fora de seu hábitat natural, é cultivada como planta ornamental em estufas na Europa e América do Norte (Prance 1974; Sculthorpe 1985) e no Brasil em lagos naturais e artificiais em coleções particulares e jardins botânicos.

As grandes flores solitárias de Victoria amazonica apresentam protoginia e são polinizadas por besouros do gênero Cyclocephala (Dynastinae) (Prance \& Arias 1975; Rosa-Osman et al. 2011). Estes besouros são atraídos pelo odor e coloração das pétalas, frequentemente se alimentando e copulando enquanto permanecem presos dentro da câmara floral de $V$. amazonica, atuando como importante polinizador da espécie (Seymour \& Matthews 2006).

Pouco se sabe sobre a ecologia em geral de Victoria amazonica fora de sua área de distribuição nativa, não havendo registro de polinização sem interferência humana nestas condições. Além disso, faltam estudos sobre a sua reprodução e manejo em condições de cultivo ex situ. Com o propósito de contribuir para o estabelecimento de estratégias que auxiliem no processo de manejo e conservação ex situ desta espécie, o presente trabalho teve por objetivo determinar a fauna de besouros presos dentro da câmara floral de $V$. amazonica cultivadas no Jardim Botânico Municipal de Bauru/SP, avaliando a eficiência desta fauna como polinizadores.

\section{Material e Métodos}

Os dados foram coletados em duas plantas de Victoria amazonica pertencentes à coleção ex situ de plantas do Jardim Botânico Municipal de Bauru (JBMB), localizado no município de Bauru/ SP, dentre os meses de fevereiro a maio de 2014 . O JBMB está inserido em um fragmento de vegetação nativa, com 321 hectares, predominantemente de Cerrado e Floresta Estacional Semidecidual, distante cerca de um quilômetro da área urbana. O clima da região é definido pela classificação Köppen-Geiger como Aw, tropical chuvoso com inverno seco (Peel et al. 2007), com temperatura média anual de $23,8^{\circ} \mathrm{C}$ (IPMET 2016). As plantas de $V$. amazonica foram mantidas em vasos com substrato, dentro de um lago artificial de $700 \mathrm{~m}^{2}$, com profundidade aproximada de 0,6 $\mathrm{m}$.

Inicialmente foram realizadas observações para determinar os horários de abertura e fechamento das flores, bem como o comportamento dos besouros sem interferência externa, no primeiro dia de abertura da flor (em torno das 19:00 h) e no segundo dia de abertura da flor (às 8:00 h e em torno das 19:00 h).

A presença de besouros foi determinada através de visualização direta da flor, sempre após o primeiro dia de abertura, por volta das 8 horas da manhã, período em que a câmara floral estava quase totalmente fechada. As flores foram abertas manualmente, afastando as pétalas entre si para visualização do interior da câmara floral. Quando presentes, fazia-se a contagem e coleta dos besouros para posterior identificação. Foram acompanhadas ao todo 20 flores ao longo do período de estudo.

Após o período de antese todos os pecíolos foram demarcados indicando a presença ou não do besouro preso na câmara floral no período de antese. No início do desenvolvimento os frutos foram cobertos com sacos de poliéster para evitar a liberação das sementes quando estivessem maduras. Com o amadurecimento e abertura espontânea dos frutos, as sementes foram coletadas, contadas e separadas aquelas a serem utilizadas nos testes de germinação.

Para determinar a viabilidade das sementes foi realizado o teste de germinação em quatro repetições com 25 sementes, escolhidas aleatoriamente. Assim que as sementes foram coletadas, o arilo foi retirado manualmente e feito a assepsia com hipoclorito de sódio ( $\mathrm{NaClO}$ ) a 1\% e posterior lavagem em água corrente. As sementes então foram incubadas em câmaras de germinação (TE-401, TECNAL, Brasil) a $29^{\circ} \mathrm{C}$ e fotoperíodo de $12 \mathrm{~h}$, dentro de tubos de ensaio contendo água. O teste de germinação foi conduzido pelo tempo necessário para a estabilização da germinação, sendo consideradas germinadas aquelas sementes em que havia protusão do eixo embrionário (Sculthorpe 1985). Os resultados foram expressos em percentagem de germinação.

\section{Resultados e Discussão}

Em nossas observações, as flores de Victoria amazonica se abrem por volta das 19:00 hs em seu primeiro dia de abertura, emitindo forte odor cítrico e apresentando pétalas brancas. Foram observados besouros abordando a flor e entrando imediatamente na abertura da câmara floral. No início da manhã do dia seguinte, a câmara floral apresentava-se fechada, permanecendo assim durante toda a manhã e tarde. Por volta das 16:00 h, as pétalas adquiram gradualmente uma coloração 
arroxeada e a partir das 19:00 hs aproximadamente, a câmara floral abriu-se lentamente, liberando os besouros. Foram observados apêndices carpelares danificados pelos besouros que, de acordo com Seymour \& Matthews (2006), alimentam-se destes apêndices. Esta sequência de antese das flores de $V$. amazonica também foi descrita por Prance \& Arias (1975), com pequenas diferenças nos horários de abertura e fechamento das flores, possivelmente relacionadas às características abióticas do ambiente (temperatura e luminosidade).

Foram visualizados besouros da espécie Cyclocephala epistomalis Bates, 1888 (Coleoptera: Scarabaeidae: Dynastinae: Cyclocephalini) (det. J. Fuhrmann 2015) presos dentro das flores, sendo o primeiro registro de interação entre esta espécie de besouro e flores de Victoria amazonica (Fig. 1a). Da mesma forma, não há relatos anteriores de que plantas de $V$. amazonica cultivadas em condições ex situ, fora da região amazônica, pudessem se reproduzir sexualmente sem interferência humana. Além disso, também é o primeiro registro desta espécie de besouro no estado de São Paulo. Os besouros coletados no interior da câmara floral possuíam pólen aderido a várias partes do corpo, o que aumenta a probabilidade de transferência de pólen para o estigma da flor (Alves-dos-Santos et al. 2016).

Gessner $(1960,1962)$ registrou a polinização de flores de Victoria amazonica exclusivamente por besouros da espécie Cyclocephala castanea
Oliv., enquanto Prance \& Arias (1975) relataram a presença de quatro espécies de besouros visitando flores desta espécie, C. castanea Oliv., C. hardyi Endrödi, C. verticalis Burmeister e Ligyrus similis Endrödi. Cyclocephala é um gênero de distribuição Neotropical, composto por inúmeras espécies muito semelhantes entre si (Saylor 1945), o que pode levar a comportamentos também semelhantes de polinização. São encontrados tanto em áreas urbanas e periurbanas, como em áreas de cerrado e floresta, frequentemente associados a polinização de espécies nativas (Gottsberger 1999; GascaÁlvarez \& Amat-García 2010; Rodrigues et al. 2010; Luçardo et al. 2014; Paulino-Neto 2014). Além disso, estas espécies pertencem a gêneros de plantas nativas que ocorrem em remanescente florestal próximo a área de estudo, possivelmente aumentando a disponibilidade destes insetos polinizadores (Gottsberger 1994; Cavalcante et al. 2009; Oliveira \& Ávila 2011; Luçardo et al. 2014; Paulino-Neto 2014; Almeida \& Louzada 2015). São besouros de hábitos noturnos, atraídos pelo odor e coloração das flores, das quais alimentam-se de pólen, partes florais e exudatos nutritivos, acabando por polinizá-las (Gottsberger 1986).

Neste estudo, foram observados um a cinco besouros por flor, um número relativamente baixo, quando comparado ao trabalho de RosaOsman et al. (2011), que observaram em média 23 besouros por flor. Como estes besouros são atraídos principalmente pelo odor e coloração das flores,
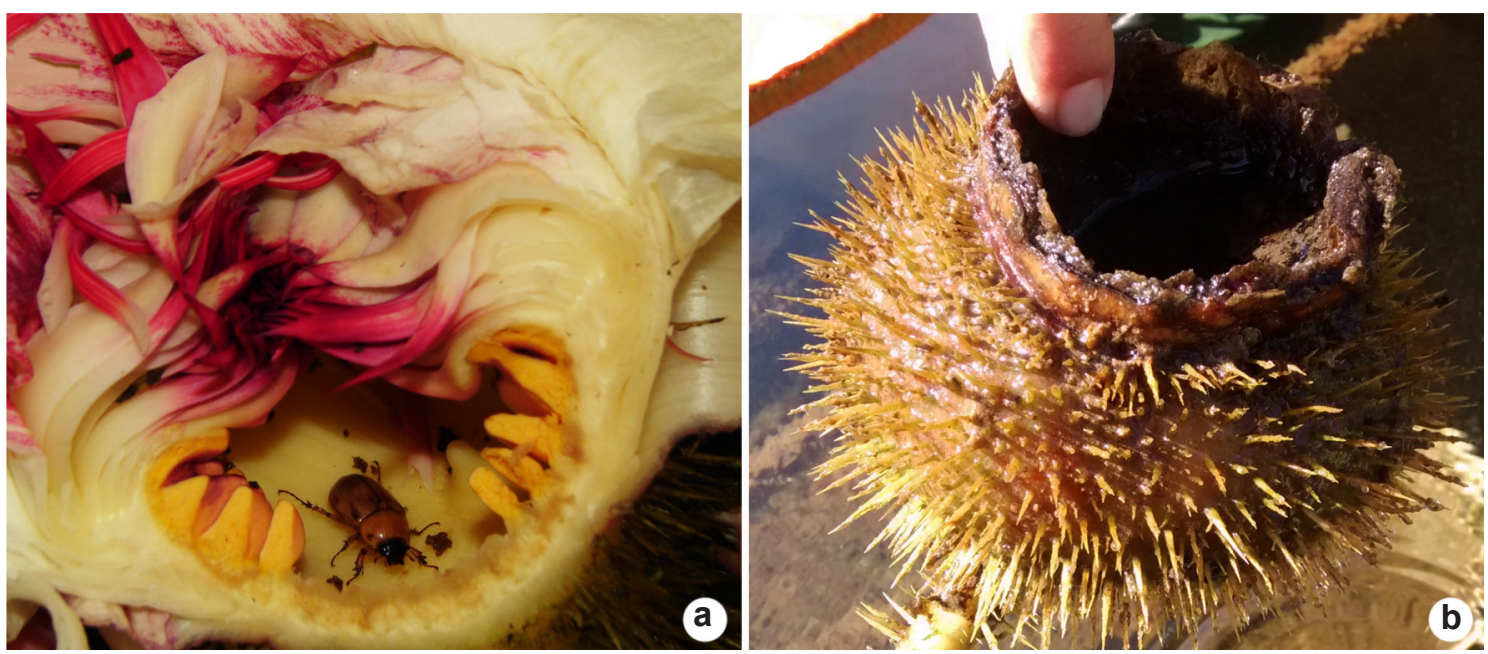

Figura 1 - a. Seção transversal da flor de Victoria amazonica (Nymphaeaceae) evidenciando o besouro da espécie Cyclocephala epistomalis no interior da câmara floral; b. fruto em formação.

Figure 1 - a. Section of Victoria amazonica (Nymphaeaceae) flowers showing the beetle Cyclocephala epistomalis in the floral chamber; b. fruit in development. 
o baixo número de flores de Victoria amazonica no presente estudo (apenas uma por vez em dois indivíduos) pode ter contribuído para uma menor quantidade de besouros presos na câmara floral, em contraste a estudos na região amazônica onde há um número grande de flores disponíveis (Prance \& Arias 1975; Rosa-Osman et al. 2011). Além disso, é possível afirmar que não ocorreu polinização cruzada no presente estudo pois, não houveram flores abertas de diferentes indivíduos no mesmo dia. Isto reforça a presença de autocompatibilidade nesta espécie (Prance \& Arias 1975). Em condições naturais, o maior número de plantas e de besouros possibilita polinização cruzada nesta espécie (Prance \& Arias 1975). No entanto, como nem sempre há flores e polinizadores em abundância em condições de cultivo ex situ, a autocompatibilidade pode facilitar a reprodução sexuada desta espécie.

Das 20 flores acompanhadas, dez não apresentaram besouros presos na câmara floral e não formaram frutos viáveis (sem sementes). Nas outras dez flores foi observada a presença de besouros presos na câmara floral, das quais nove produziram frutos viáveis (Fig. 1b), com grande quantidade de sementes $(335,88 \pm 53,11$ sementes por fruto) e uma produziu fruto com apenas uma semente. Os resultados reforçam o papel do besouro na polinização de flores desta espécie e apontam para a presença de besouros na câmara floral como determinante na formação de frutos viáveis. Estudos anteriores na região amazônica encontraram em média 328 sementes por fruto (Prance \& Arias 1975) e 114 sementes por fruto (Rosa-Osman et al. 2011). Seymour \& Matthews (2006) sugerem maior fecundação quando há maior número de besouros presos dentro da câmara, o que pode estar relacionado à variação no número de sementes entre os diferentes estudos. Rosa-Osman et al. (2011), no entanto, relacionaram a grande quantidade de coleópteros com a predação de óvulos e consequente baixa quantidade de sementes em frutos de Victoria amazonica.

O tempo de maturação dos frutos advindos de flores nas quais haviam besouros presos foi de 5065 dias, quando liberaram espontaneamente suas sementes. A germinação das sementes iniciou-se a partir do $9^{\circ}$ dia e o pico máximo ocorreu no $21^{\circ}$ dia ( $88 \%$ das sementes germinadas), a partir do qual nenhum incremento na germinação foi observado.

Em razão do comportamento dos besouros na entrada das flores, da permanência destes dentro da câmara floral, do elevado número de sementes por fruto e pela elevada taxa de germinação das sementes, observados neste estudo, pode-se inferir que os besouros comportaram-se como polinizadores efetivos e que a polinização desta espécie é possível também em condições de cultivo ex situ, sem interferência humana, onde existam polinizadores. Estes resultados podem auxiliar em estudos de manejo desta espécie ex situ, permitindo ampliar as estratégias que possibilitam a sua propagação nestas condições. Nossos resultados trazem evidências também do potencial de Victoria amazonica em colonizar novos ambientes onde existam polinizadores, podendo tornar-se uma espécie subespontânea a espontânea. No entanto, há de se considerar que estas conclusões foram tomadas a partir da observação de apenas dois indivíduos em um curto período de tempo, sendo necessários mais estudos utilizando maior número de plantas e que abordem outras dimensões da biologia desta espécie e suas interações com fatores bióticos e abióticos.

\section{Referências}

Almeida SSP \& Louzada JNC (2015) Estrutura da comunidade de Scarabaeinae (Scarabaeidae: Coleoptera) em fitofisionomias do cerrado e sua importância para a conservação. Neotropical Entomology 38: 32-43.

Alves-dos-Santos I, Silva CI, Pinheiro M \& Kleinert AMP (2016) Quando um visitante floral é um polinizador? Rodriguésia 67: 295-307.

BFG - The Brazil Flora Group (2015) Growing knowledge: na overview of seed plant diversity in Brazil. Rodriguésia 66: 1085-1113.

Cavalcante TM, Naves RV, Franceschinelli EV \& Pereira-da-Silva R (2009) Polinização e formação de frutos em Araticum. Bragantia 68: 13-21.

Gasca-Álvarez HJ \& Amat-García G (2010). Synopsis and key to the genera of Dynastinae (Coleoptera, Scarabaeoidea, Scarabaeidae) of Colombia. ZooKeys 34: 153:192.

Gessner F (1960) The opening of flowers of Victoria regia in dependence of light. Planta 54: 453-463.

Gessner F (1962) The opening of flowers of Victoria regia in relation the light. Boletim do Museu Emilio Goeldi, Botânica 17: 1-13.

Gottsberger G (1986) Some pollinattion strategies in neotropical savanas and forests. Plant Systematics and Evolution 152: 29-45.

Gottsberger G (1994) As anonáceas do cerrado e a sua polinização. Revista Brasileira Biologia 54: 391-402.

Gottsberger G (1999) Pollination and evolution in neotropical Annonaceae. Plant Species Biology 14: 143-152. 
IPMet (2016) Estação meteorológica automática. Disponível em $<$ http://www.ipmet.unesp.br/index2. php? menu_esq1=\&abre=ipmet_html/estacao/ historico.php> Acesso em 12 abril 2016.

Judd WS, Campbell CS, Kellogg EA, Stevens PF \& Donoghue MJ (2002) Plant systematics: a phylogenetic approach. Sinauer Associates, Sunderland. 576p.

Luçardo M, Oliveira CM \& Frizzas MR (2014) Scarabaeidea (Insecta: Coleoptera) no cerrado brasileiro: estado atual do conhecimento. Ciência Rural 44: 652-659.

Oliveira HN \& Ávila CJ (2011) Ocorrência de Cyclocephala forsteri em Acrocomia aculeata. Pesquisa Agropecuária Tropical 41: 293-295.

Paulino-Neto HF (2014) Polinização e biologia reprodutiva de Araticum-liso (Annona coriacea Mart.: Annonaceae) em uma área de cerrado paulista: implicações para fruticultura. Revista Brasileira de Fruticultura 36: 132-140.

Peel MC, Finlayson BL \& Mcmahon TA (2007) Update world map of the Köppen-Geiger climate classification. Hydrology and Earth System Science 11: 1633-1644.

Pott VJ \& Pott A (2000) Aquatic plants of Pantanal. Embrapa, Brasília. 404p.
Prance GT (1974) Victoria amazonica or Victoria regia? Acta Amazonica 4: 5-8.

Prance GT \& Arias JR (1975) A study of the floral biology of Victoria amazonica (Poepp.) Sowerby (Nymphaeaceae). Acta Amazonica 5: 109-139.

Rodrigues SP, Nogueira GAL, Echeverria RR \& Oliveira VS (2010) Aspectos da biologia de Cyclocephala verticalis Burmeister (Coleoptera: Scarabaeidae). Neotropical Entomology 39: 15-18.

Rosa-Osman SM, Rodrigues R, Mendonça MS, Souza LA \& Piedade MTF (2011) Morfologia da flor, fruto e plântula de Victoria amazonica (Poepp.) J.C. Sowerby (Nymphaeaceae). Acta Amazonica 41: 21-28.

Saylor LW (1945) Synoptic revision of the United States scarab beetles of the subfamily Dynastinae, $n^{\circ} .1$ : tribe Cyclo-Cephalini. Journal of the Washington Academy of Science 35: 378-386.

Sculthorpe CD (1985) The biology of aquatic vascular plants. Edward Arnold Publishers, London. 610p.

Seymour RS \& Matthews PGD (2006) The role of thermogenesis in the pollination biology of the amazona waterlily Victoria amazonica. Annals of Botany 98: 1129-1135. 